\title{
Régimes thermiques de sols de l'étage périglaciaire et leurs conséquences géomorphologiques
}

Exemple de la combe de Laurichard, Alpes du Briançonnais, France

\section{Temperature Fluctuations in Alpine Bedrocks and their Geomorphic Consequences}

\author{
Exemple of the Combe de Laurichard (Alps of the Briançonnais)
}

\section{Temperatursch-wingungen in alpinem Muttergestein und ihre geomorphologischen Folgen}

\author{
Beispiel des Combe de Laurichard (Briançonnaise Alpen)
}

\section{Bernard Francou}

Volume 37, numéro 1, 1983

URI : https://id.erudit.org/iderudit/032496ar

DOI : https://doi.org/10.7202/032496ar

Aller au sommaire du numéro

\section{Éditeur(s)}

Les Presses de l'Université de Montréal

ISSN

0705-7199 (imprimé)

1492-143X (numérique)

Découvrir la revue

Citer cet article

Francou, B. (1983). Régimes thermiques de sols de l'étage périglaciaire et leurs conséquences géomorphologiques : exemple de la combe de Laurichard, Alpes du Briançonnais, France. Géographie physique et Quaternaire, 37(1), 27-38. https://doi.org/10.7202/032496ar

\section{Résumé de l'article}

En utilisant les température mesurées dans le sol en 4 sites entre $2450 \mathrm{~m}$ et $2650 \mathrm{~m}$ dans la combe de Laurichard, on cherche avec l'appoint des températures sous abri et les relevés effectués sur les hauteurs de neige à analyser les conditions dans lesquelles le froid hivernal s'accumule dans les sols d'éboulis jusqu'à $-50 \mathrm{~cm}$ et celles qui provoquent son expulsion pendant l'été. C'est la neige qui règle directement l'évolution des températures dans le sol, mais son rôle isolant n'est pas, notamment sur les versants d'éboulis, aussi absolu qu'on a pu le penser. Le décalage du manteau neigeux par rapport au minimum thermique de décembre-février peut favoriser certaines années un engel accentué des éboulis d'ubac et des topographies ventées du fond de la combe. En adret, pendant l'hiver, la couche de neige ne paivient pas à maintenir durablement une épaisseur qui empêche les variations thermiques courtes de la surface de se répercuter, bien que très amorties, en profondeur; inversement, le maintien du manteau neigeux, au début de l'été, tend à prolonger une homothermie faiblement négative dans le sol. On conclut qu'il est difficile, mais pas impossible selon la topographie, qu'un pergélisol puisse s'établir dans des sites d'exposition nord-ouest ; cependant, pour expliquer la présence actuelle de glace dans les matériaux mobiles de ces versants (glaciers rocheux et éboulis fluants), il convient d'envisager d'autres origines, jusqu'à ce que d'autres mesures en cours permettent des conclusions plus nettes.
Tous droits réservés @ Les Presses de l'Université de Montréal, 1983
Ce document est protégé par la loi sur le droit d'auteur. L’utilisation des services d'Érudit (y compris la reproduction) est assujettie à sa politique d'utilisation que vous pouvez consulter en ligne.

https://apropos.erudit.org/fr/usagers/politique-dutilisation/ 


\section{RÉGIMES THERMIQUES DE SOLS DE L'ÉTAGE PÉRIGLACIAIRE ET LEURS CONSÉQUENCES GÉOMORPHOLOGIQUES Exemple de la combe de Laurichard, Alpes du Briançonnais, France}

Bernard FRANCOU, Institut français d'études andines, Casilla 278, Lima 18, Pérou.

RÉSUMÉ En utilisant les température mesurées dans le sol en 4 sites entre $2450 \mathrm{~m}$ et $2650 \mathrm{~m}$ dans la combe de Laurichard, on cherche avec l'appoint des températures sous abri et les relevés effectués sur les hauteurs de neige à analyser les conditions dans lesquelles le froid hivernal s'accumule dans les sols d'éboulis jusqu'à $-50 \mathrm{~cm}$ et celles qui provoquent son expulsion pendant l'été. C'est la neige qui règle directement l'évolution des températures dans le sol, mais son rôle isolant n'est pas, notamment sur les versants d'éboulis, aussi absolu qu'on a pu le penser. Le décalage du manteau neigeux par rapport au minimum thermique de décembrefévrier peut favoriser certaines années un engel accentué des éboulis d'ubac et des topographies ventées du fond de la combe. En adret, pendant I'hiver, la couche de neige ne parvient pas à maintenir durablement une épaisseur qui empêche les variations thermiques courtes de la surface de se répercuter, bien que très amorties, en profondeur; inversement, le maintien du manteau neigeux, au début de l'été, tend à prolonger une homothermie faiblement négative dans le sol. On conclut qu'il est difficile, mais pas impossible selon la topographie, qu'un pergélisol puisse s'établir dans des sites d'exposition nord-ouest; cependant, pour expliquer la présence actuelle de glace dans les matériaux mobiles de ces versants (glaciers rocheux et éboulis fluants), il convient d'envisager d'autres origines, jusqu'à ce que d'autres mesures en cours permettent des conclusions plus nettes.
ABSTRACT Temperature fluctuations in Alpine bedrocks and their geomorphic consequences. Exemple of the Combe de Laurichard (Alps of the Briançonnais). Using ground temperatures measured at 4 sites between $2450 \mathrm{~m}$ and $2650 \mathrm{~m}$ in the Combe de Laurichard, we attempted to analyse, with the additional data of screen temperatures and snow depth, the conditions controlling the annual accumulation of frost in the scree materials down to a depth of $50 \mathrm{~cm}$ during the winter and its expuision during the summer. Temperature fluctuations are directly controlled by snow cover. However, thermal insulation may not be as efficient as it may seems, especially in talus slopes. During some winters, late development of snow cover after minimum temperatures are reached can cause severe freezing in northern slope screes and windy valley bottoms. On the southern slope, snow cover may not be thick enough to prevent short thermal fluctuations from the surface, although they are minimal in depth. On the other hand, persistance of the snow cover until the beginning of summer tends to maintain freezing temperatures in the ground. We reach to the conclusion that it is almost impossible for permafrost to develop in northwestern screes, except in micro-environments. Consequently, other explanations for the presence of ice in these materials (rock glaciers and flowing screes) have to be considered, until new data are provided giving more conclusive answers.
ZUMMENFASSUNG Temperaturschwingungen in alpinem Muttergestein und ihre geomorphologischen Folgen. Beispiel des Combe de Laurichard (Briaçonnaise Alpen). Bodentemperaturen gebrauchend, die an vier Orten zwischen $2450 \mathrm{~m}$ und $2650 \mathrm{~m}$ in Combe de Laurichard gemessen wurden, haben wir, mit Hilfe von zusätzlichen Daten wie Temperaturen und Schneetiefe, versucht die Bedingungen, welche die jährliche Ansammlung von Frost bis zu $50 \mathrm{~cm}$ Tiefe im Material der Schuttanhäufungen verursacht, zu analysieren, sowie dessen Ausstoss im Sommer. Die Temperaturschwingungen werden durch die Schneedecke kontroliert. Jedoch, kommt es vor, dass die Wärmeisolierung nicht so wirksam ist, wie es scheint, besonders an den Talhängen. In einigen Wintern, kann späte Entwicklung der Schneedecke nachdem die Tiefsttemperaturen schon erreicht wurden, schweres Gefrieren der Schuttanhäufungen an nördlichen Hängen und in windigen Talböden verursachen. An Südhängen, kann die Schneedecke zu dünn sein, um kurze thermische Schwingungen an der Oberfläche zu verhindern, wenn sie auch minimal an Tiefe bleiben. Andererseits hält das Bestehen der Schneedecke bis zu Beginn des Sommers die Frosttemperaturen im Boden fest. Wir kommen zu der Schlussfolgerung, dass es fast unmöglich ist, dass sich Dauerfrost in den nordwestlichen Schuttanhäufungen entwickelt, unter Ausschluss von Mikro-Umgebungen. Infolgedessen müssen andere Erklärungen für die Anwesenheit von $\mathrm{E}$ is in diesen Materialien (Felsgletscher und fliessende Schuttanhäufungen) erwägt werden, bis neue Daten mit beweiskräftigeren Antworten gefunden sind. 


\section{LE TERRAIN D'ÉTUDE}

La présente étude concerne les Alpes françaises, plus particulièrement la zone intra-alpine briançonnaise. Cette région est connue par sa luminosité et sa sécheresse relative responsables de contrastes thermiques importants et de la remontée en altitude de la limite des neiges permanentes; celle-ci se situe entre $2900 \mathrm{~m}$ et $3200 \mathrm{~m}$ selon l'exposition, et l'étage périglaciaire proprement dit se développe au-dessous jusque vers $2500 \mathrm{~m}$, altitude où stationne l'isotherme annuelle $0^{\circ} \mathrm{C}$ et à laquelle les pelouses et les landes alpines commencent à coloniser les pentes.

Le modelé de cet étage est dominé par les actions cryonivales, mais aussi par une convergence de processus favorisant un stockage important de la glace dans les sols, assurant ainsi le fonctionnement de nombreux "éboulis fluants" et de glaciers rocheux d'origine non exclusivement glaciaire (FRANCOU, 1981 ; JORDA, 1977).

On connaît encore mal l'évolution tout au long de l'année des températures des sols à cette altitude, particulièrement dans les secteurs dénudés de végétation couverts par des nappes d'éboulis. En présentant les premiers résultats de mesures effectuées à $50 \mathrm{~cm}$ de profondeur dans quatre sites, cette étude vise à mieux délimiter les influences relatives de l'exposition, de la pente, du manteau neigeux et de la granulométrie sur les échanges thermiques entre l'air et le sol.

Le site étudié, depuis octobre 1979, est celui de la combe de Laurichard, qui est un vallon encaissé et froid sur le versant nord du massif du Combeynot $(3110 \mathrm{~m})$ débouchant sur le col du Lautaret accessible toute l'année; il constitue le cadre privilégié d'une série d'études portant sur les dynamiques actuelles des versants de la haute montagne et concernant plus spécialement le démantèlement des parois, les chutes de pierres (FRANCOU, 1981), l'éboulisation et son rapport avec les glaciers rocheux (FRANCOU, 1981, 1983). Trois principaux micro-milieux sont représentés dans la combe (fig. 1): a) un grand versant ubac (NO) de $650 \mathrm{~m}$ de dénivelée dont $400 \mathrm{~m}$ de paroi rocheuse raide culminant à $3100 \mathrm{~m}$, avec de beaux névés persistants au pied; b) un versant adret (SE) plus court, de $350 \mathrm{~m}$, formant la Pyramide de Laurichard à $2770 \mathrm{~m}$ où apparaissent déjà, par taches, de nombreux orophytes; c) un fond non drainé à $2450 \mathrm{~m}$ subissant fortement le masque orographique encadrant et portant une lande très lacunaire. $\mathrm{Ce}$ sont ces trois milieux qui fournissent la base de la présente étude comparative sur les micro-environnements thermiques.

\section{L'INSTRUMENTATION ET LES SITES DE MESURES}

1. Au fond de la combe, la station de base (B) installée sur une topographie bosselée et un sol arénacé emballant de nombreux blocs permet de mesurer: a) les températures sous abri météorologique; b) les températures dans le sol à $-10 \mathrm{~cm}$ et $-50 \mathrm{~cm}$, en continu sur un replat déprimé sur-alimenté en neige, par prises périodiques avec deux pyrosondes placées sur un replat venté en position culminante; c) la hauteur du manteau neigeux en trois points P3, P4, P5, les deux premiers sur le site des thermosondes (P3) et des pyrosondes (P4), le dernier sur une bosse très vite déneigée (P5). Les deux sols comportent un horizon humifère mince de $20 \mathrm{~cm}$ et sont médiocrement drainés sans être hydromorphes.

2. En adret, la station (A1) est à $2550 \mathrm{~m}$ au sommet d'un talus d'éboulis, à $4 \mathrm{~m}$ de la paroi rocheuse, et elle comprend: a) une thermosonde à deux capteurs placés à $-50 \mathrm{~cm}$, l'un dans des blocs décimétriques en open-work, l'autre dans des graviers à matrice surtout sableuse; b) un thermomètre à maxi-minima sous abri. L'éboulis est peu actif, en voie de colonisation végétale par le haut, mais le site, lui, est sans végétation.

3. En ubac, l'appareillage est installé au sommet d'un talus d'éboulis (U1), en lisière d'un couloir, et regroupe à $2650 \mathrm{~m}:$ a) une thermosonde à deux capteurs à $3 \mathrm{~m}$ de la paroi, le premier à $-20 \mathrm{~cm}$ sous des blocs en open-work, le second à $-50 \mathrm{~cm}$ dans I'horizon sousjacent enrichi en fraction fine éluviée, en majorité sableuse; b) un thermomètre à maxi-minima en position d'abri dans la paroi. L'éboulis est très actif, sans végétation, mais sans fluage apparent, fonctionnant comme un cône d'avalanche mixte.

A 50 mètres à l'ouest, existe un névé permanent recouvert de gélifracts en fin d'été, où prend naissance un glacier rocheux actif; une rimaye thermique ouverte au contact de la paroi a pu donner, à l'aide d'une thermosonde enfoncée à $-500 \mathrm{~cm}$ en terme de fusion, quelques mesures fragmentaires au cours des deux années.

Les données fournies ici par des thermographes Richard-Pékly ont été constamment vérifiées à l'aide des pyrosondes placées en parallèle et leur fiabilité est correcte dans la limite de $1,5^{\circ} \mathrm{C}$. Elles sont continues et regroupées en périodes de dix jours pour les thermographes, d'une périodicité peu différente du mois pour les mini-maxima, les pyrosondes et les hauteurs de neige.

On a choisi l'année 1980-81 centrée sur l'hiver présentant les mesures les plus sûres, mais on a étendu l'échantillonnage à l'année 1979-80 en ce qui concerne les amplitudes thermiques et quelques relevés de températures dans les sols. II s'agit là de premiers éléments pour des analyses qui devront être développées à partir de mesures plus longues et plus variées.

\section{LE RÉGIME DU FROID DANS L'AIR ET LA DURÉE DU MANTEAU NEIGEUX}

1. Le gel est présent pendant près des deux tiers de l'année. La température moyenne de l'air en 1980 a 


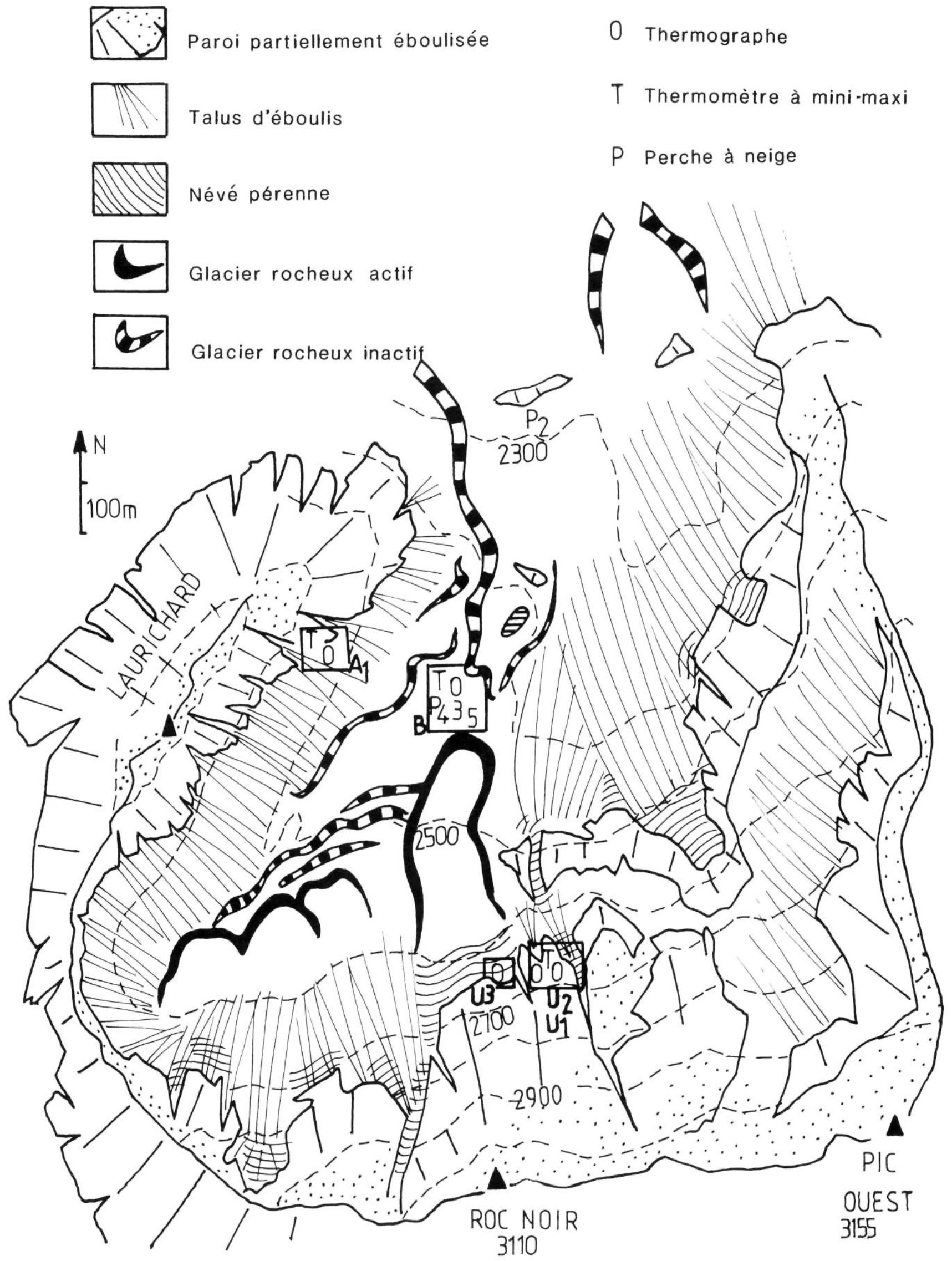

FIGURE 1. La combe de Laurichard: croquis de situation.

The Combe de Laurichard: map of studied area. 
atteint au fond de la combe $(2450 \mathrm{~m})-0,25^{\circ} \mathrm{C}$ (entre $1,9^{\circ} \mathrm{C}$ et $-2,4^{\circ} \mathrm{C}$ ), ce qui place l'isotherme $0^{\circ} \mathrm{C}$ à $2400 \mathrm{~m}$, conformément aux années moyennes des autres stations du Briançonnais ${ }^{1}$.

a) Les jours sans dégel sont très majoritaires, 168, soit $46 \%$ de l'année; en décomposant, on trouve que les longues séries à gel continu dominent largement: - gel sans dégel sur plus de 10 jours consécutifs $77,3 \%$. - gel sans dégel de 2 à 10 jours consécutifs . . . . 18,4\%. - gel sand dégel pendant une seule journée . . . . 4,3\%. Ainsi sont réunies de bonnes conditions d'engel profond des sols, d'autant plus que ce sont les séries longues qui enregistrent les gels les plus intenses: on sait en effet, par le laboratoire, que la pénétration de l'onde de gel vers $-50 \mathrm{~cm}$ suppose une continuité des températures négatives pendant au moins deux semaines, avec $-10^{\circ} \mathrm{C}$. (LAUTRIDOU, 1971). Toutefois, si les maxima moyens restent négatifs de novembre à mars, les valeurs extrêmes de froid atteintes sur deux ans ont paru faibles : $-17^{\circ} \mathrm{C}$ et $-18^{\circ} \mathrm{C}$ pour cette station.

b) Les jours à gel-dégel sont moins nombreux dans ce fond de vallon, 80 soit $22 \%$ de l'année, mais ajoutés aux jours sans dégel, ils accentuent l'écrasante présence du froid.

c) Les jours sans gel sont minoritaires (32\%), mais encore plus groupés que les premiers:

- longues séries continues (plus de 10 jours) . . .81,1\%. - séries moyennes continues (2 à 10 jours) . . . . . 17,1\%. - jours sans gel isolés ..................

Dans les conditions optimales (août), les minima moyens atteignent $+10^{\circ} \mathrm{C}$ et les maxima absolus dépassent $+20^{\circ} \mathrm{C}$ (fig. 2 et 3 ). Aussi existe-t-il de bonnes conditions de dégel profond des sols, surtout sur les versants, où les maxima sont nettement redressés par rapport au fond, même à l'ubac (fig. 3). Mais le manteau neigeux altère profondément les échanges thermiques entre l'air et le sol.

2. Le manteau neigeux est présent dans le site 9 mois sur 12. Il est observé au sol pendant tous les mois à température moyenne négative, mais il atteint son épaisseur maximale seulement après les grands froids.

a) Il est connu que l'effet isolant de la neige dépend principalement de l'épaisseur de la couche, la décroissance de l'énergie solaire y suivant à peu près une loi exponentielle (LLIBOUTRY, 1965); les oscillations diurnes disparaissent entre $30 \mathrm{~cm}$ et $50 \mathrm{~cm}$; par contre, les ondes principales liées à des successions de types de temps pénètrent jusqu'à un mètre (THORN, 1980; MUNN, 1966).

1. Température calculée selon la formule $T m=1 / 2$ tmin. + tmax. ; en fait la vraie moyenne se situe un peu en dessous si l'on fait intervenir le facteur durée, les heures dans la journée où les températures sont proches du maximum étant moins nombreuses que celles qui sont proches du minimum (rapport de 1 à 3 sur le site). b) Le manteau neigeux s'est maintenu au sol du 8 octobre à la fin juin avec un maximum d'épaisseur voisin de $150 \mathrm{~cm}$ en avril, soit pendant plus de 8 mois en 1980-81 (contre 9 mois et $220 \mathrm{~cm}$ en 1979-80, qui est une année de référence de fort enneigement). Dans le détail, on remarque d'une part que la couche est restée inférieure ou proche du mètre pendant les mois les plus froids à cause du déficit des chutes et d'une intense déflation (fig. 4); d'autre part que les variations horizontales de l'épaisseur du manteau sont considérables selon la topographie, comme on l'a mesuré sur les trois points de mesures P3, P4, P5, voisins de quelques mètres: (1) pour la moyenne des 12 mesures, le rapport est de 1 à 8 entre $\mathrm{P} 3$ et $\mathrm{P} 5$, de 1 à 2,5 entre P4 et P5; (2) la disparition complète de la couche intervient un mois et demi plus tôt sur les bosses que dans les creux; (3) en adret, sous le sommet du talus, le manteau peut s'interrompre en plein hiver à cause de la fusion (fig. 4) et sa disparition totale est acquise avec un mois d'avance sur le fond de la combe; (4) en ubac, l'ouverture du manteau se produit en hiver sur les surfaces en relief (éboulis bombés et glaciers rocheux) par déflation, mais la couverture n'est pas sérieurement entamée avant le début de juin.

Si la neige contrôle effectivement le régime thermique du sol pendant les trois mois du maximum centré autour d'avril, la lenteur de la constitution du manteau au début de l'hiver en période froide (remarquable en 1980-81) et sa forte ablation sur les surfaces ventées avant sa consolidation printanière peuvent se combiner pour rendre possible l'engel profond des sols. II reste à savoir si c'est le cas à partir des données de nos stations, en examinant successivement les amplitudes thermiques, l'engel et le dégel.

\section{LES AMPLITUDES THERMIQUES A $-50 \mathrm{CM}$ DANS LES SOLS EN QUATRE SITES}

1. Au fond de la combe (fig. 2), à $-50 \mathrm{~cm}$ dans le sol de pelouse du point P3, les amplitudes diurnes n'apparaissent plus, les variations étant seulement liées à des séquences successives de types de temps avec un décalage de quelques jours. $A-10 \mathrm{~cm}$ (données non publiées ici), les amplitudes diurnes varient de $4^{\circ} \mathrm{C}$ à $12^{\circ} \mathrm{C}$ en saison chaude avec une latence d'une heure ou deux, mais s'annulent rapidement avec $30 \mathrm{~cm}$ de neige. $\hat{A}-50 \mathrm{~cm}$ enfin, on a pu vérifier que les variations thermiques enregistrées dans l'air sur plusieurs jours se répercutent jusqu'à un mètre de neige, mais pas audelà (homothermie complète à partir de janvier).

2. En ubac, le manteau sur le site U1 ne dépasse pas un mètre tout I'hiver, tombant même en dessous de $30 \mathrm{~cm}$ en janvier par suite de la déflation. L'amplitude diurne est totalement interceptée dans le sol avant $-20 \mathrm{~cm}$ de novembre à la mi-mars, mais l'évolution thermique générale se répercute en profondeur: d'une façon échelonnée dans le temps pendant l'engel, mais 


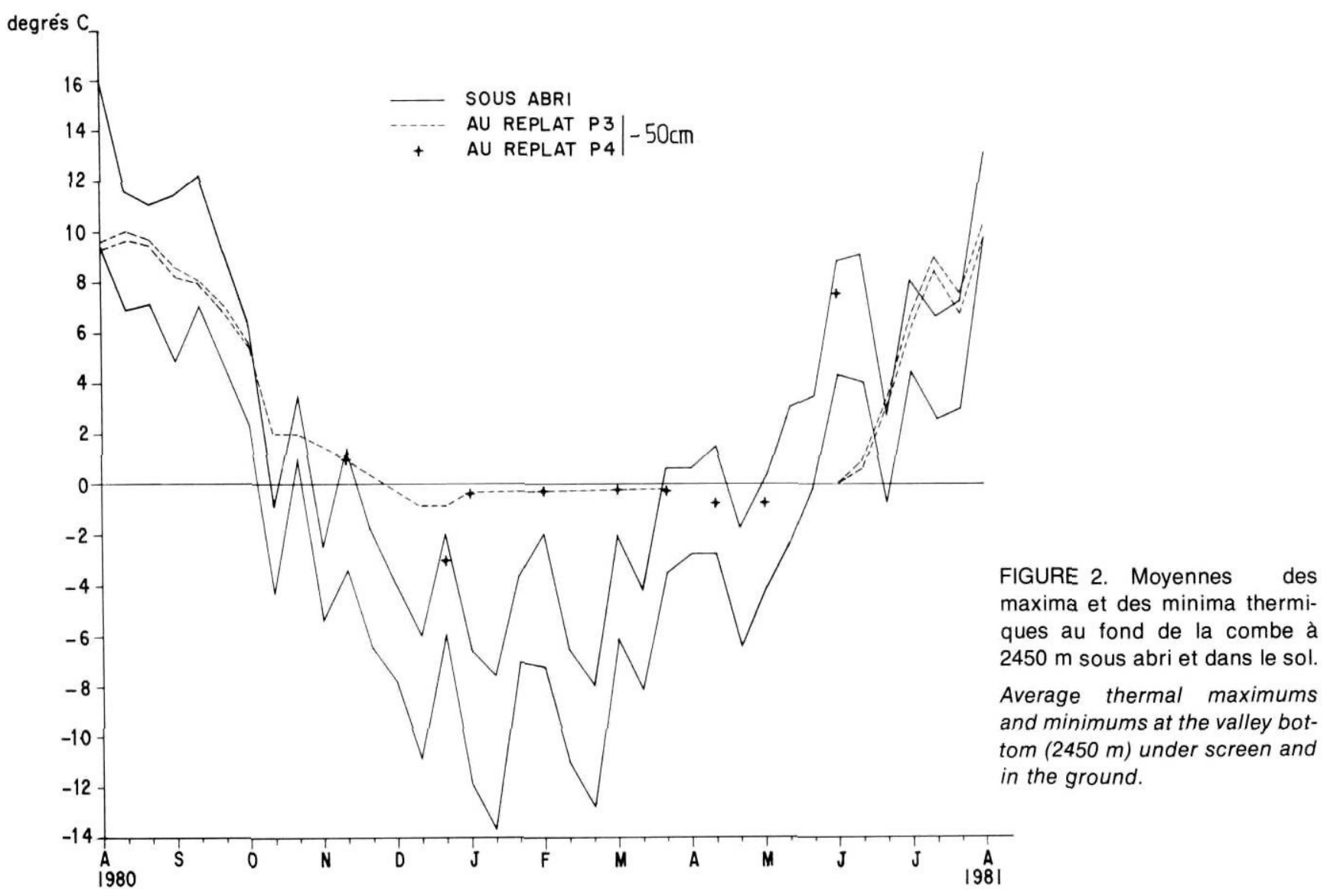

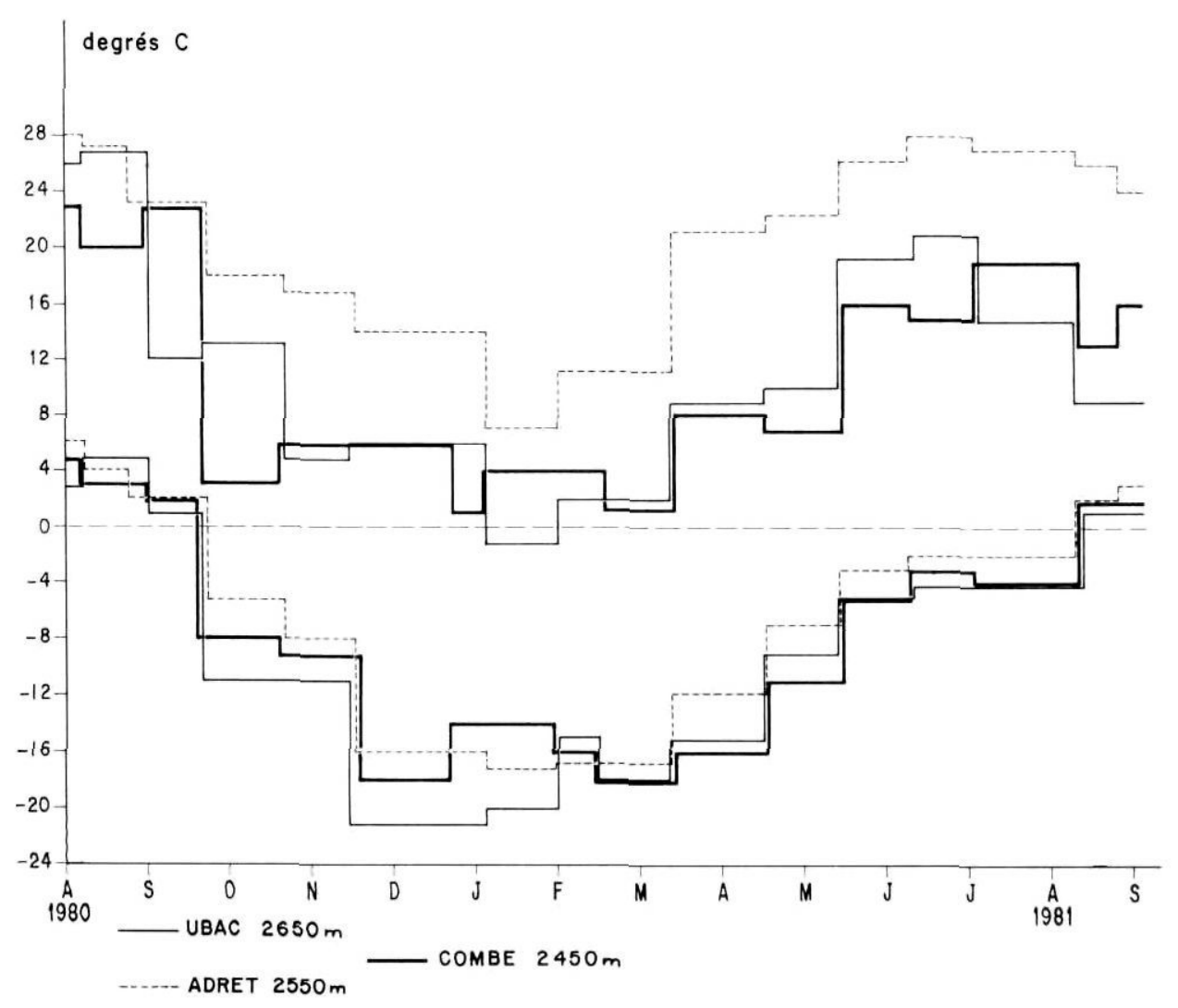

FIGURE 3. Maxima-minima absolus sous abri en trois stations.

Absolute thermal maximums and minimums at three stations under screen. 
immédiatement au moment du dégel (fig. 5 et 6 ). A la disparition du manteau, l'amplitude diurne atteint sa valeur maximale, $2^{\circ} \mathrm{C}$ environ, soit une valeur plus de trois fois inférieure à celle qui est atteinte dans l'éboulis à blocs de l'adret. On notera qu'à partir de juillet, en 1981 (août en 1980), les amplitudes sont plus fortes en profondeur $(-50 \mathrm{~cm})$ qu'à $-20 \mathrm{~cm}$, alors que la disparition du manteau devrait favoriser les échanges turbulents dans les blocs sans matrice de la surface. Alors que la température de la surface atteint son plus haut niveau (moyenne de $10^{\circ} \mathrm{C}$, maxima de $20^{\circ} \mathrm{C}$ ), ni la dynamique de l'air ni la conduction des matériaux ne peuvent expliquer cette inversion: il semble que ce soient les eaux de fusion venant de la paroi chaude qui percolent dans l'horizon inférieur et contrôlent sa température diurne. On peut fournir deux preuves à l'appui de cette hypothèse: a) au cours de la journée le maximum en profondeur se place avant celui de l'horizon $-20 \mathrm{~cm}$; b) les amplitudes des deux niveaux s'alignent de nouveau dès que la fusion dans la paroi se ralentit (août 80 ) et l'homothermie s'installe plus vite à $-50 \mathrm{~cm}$ à l'arrivée de la neige.

3. En adret, où seules, des mesures à $-50 \mathrm{~cm}$ sont disponibles (fig. 7), on note d'emblée une nette diffé-

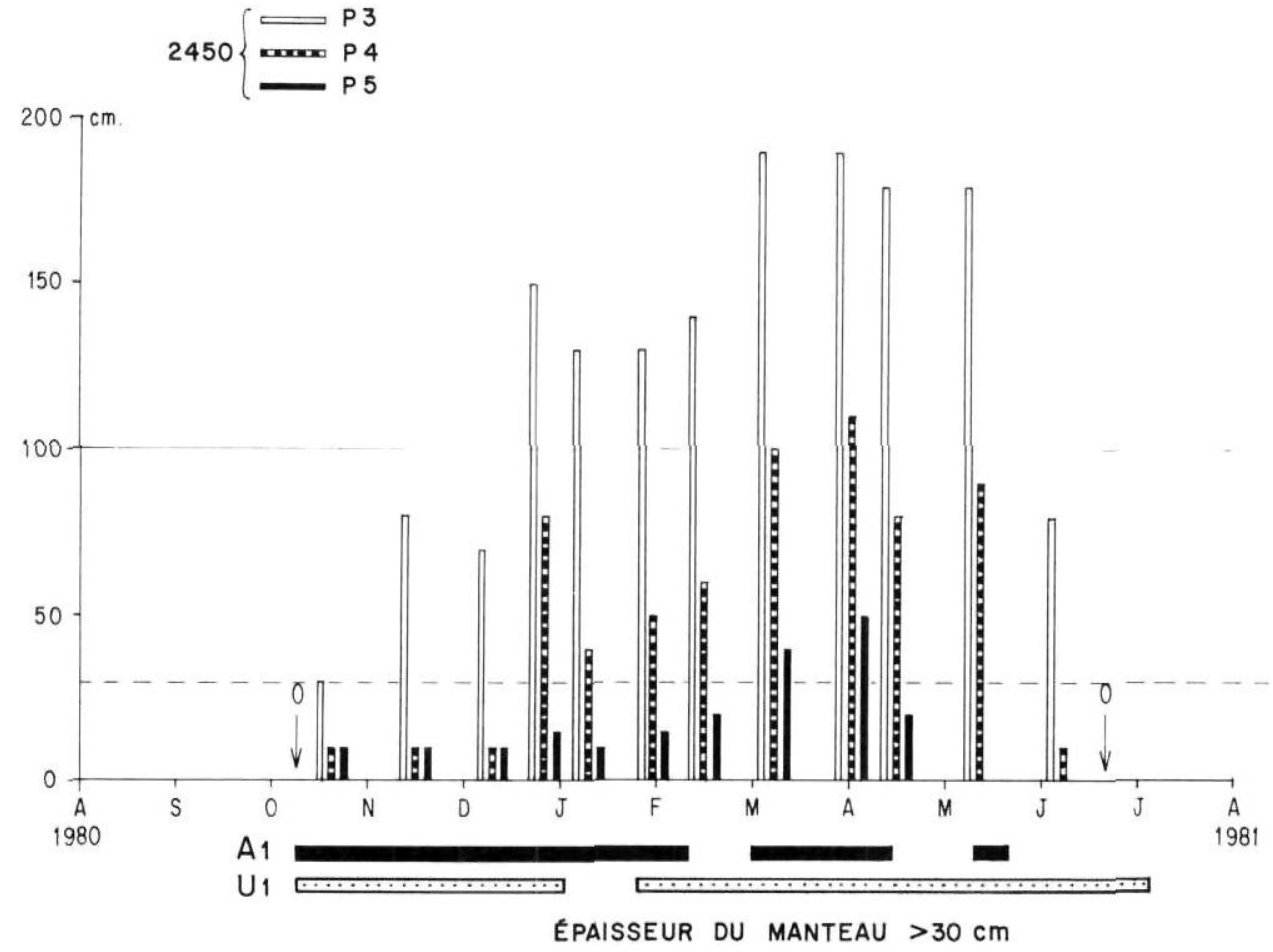

FIGURE 4. Évolution du manteau neigeux dans la combe (hiver 1980-81).

Evolution of snow cover in the valley (winter 1980-81).

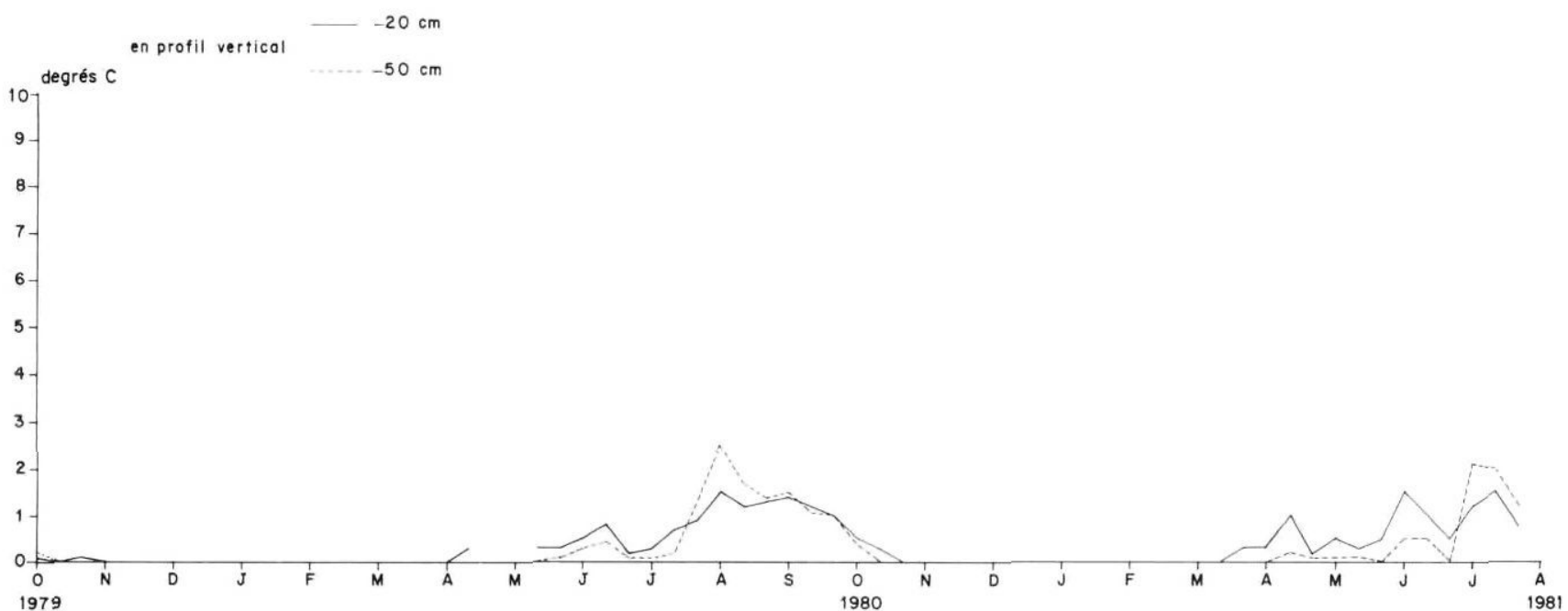

FIGURE 5. Amplitudes thermiques moyennes en ubac $(2650 \mathrm{~m})$ Average thermic ranges on the northern slope (2650 $\mathrm{m})$ in dans le sol. the ground. 


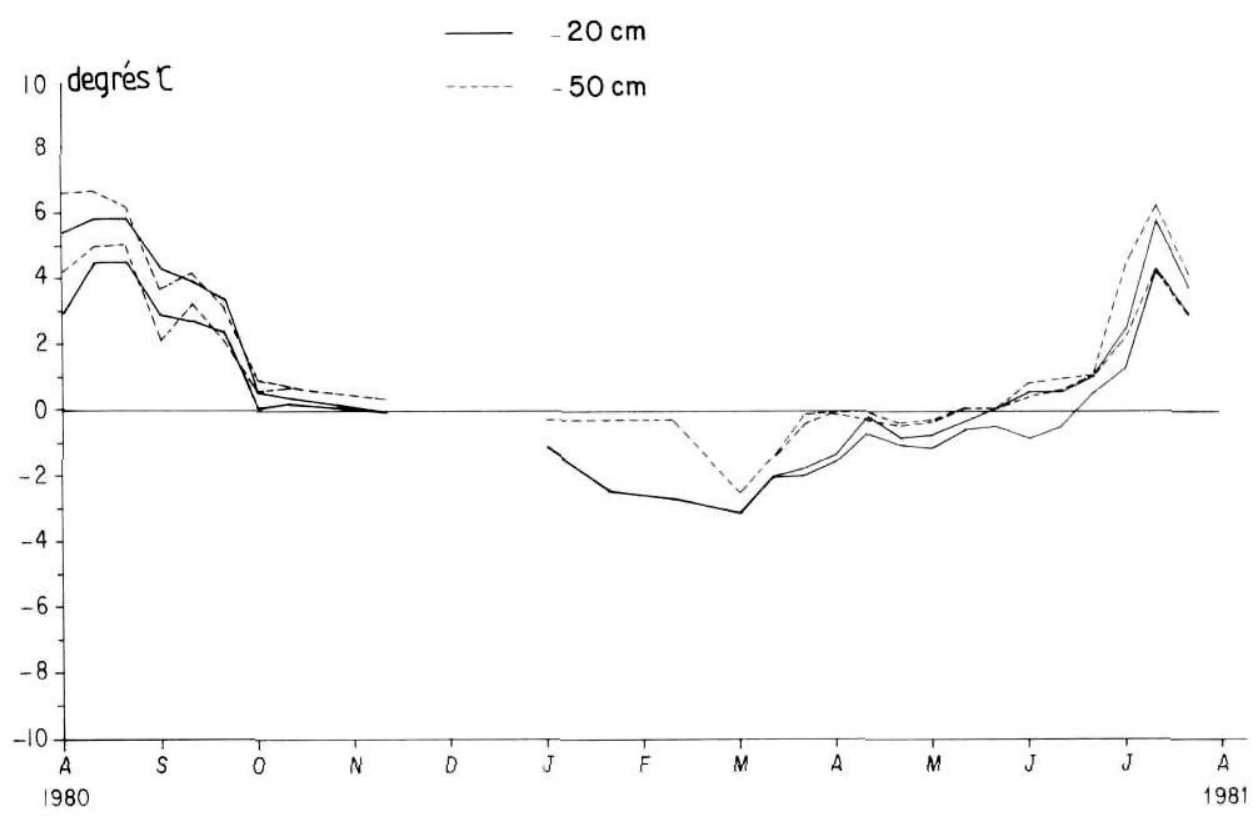

FIGURE 6. Moyennes des maxima et des minima en ubac (2650 m) dans le sol.

Mean maximum and minimum temperatures on the northern slope $(2650 \mathrm{~m})$ in the ground.

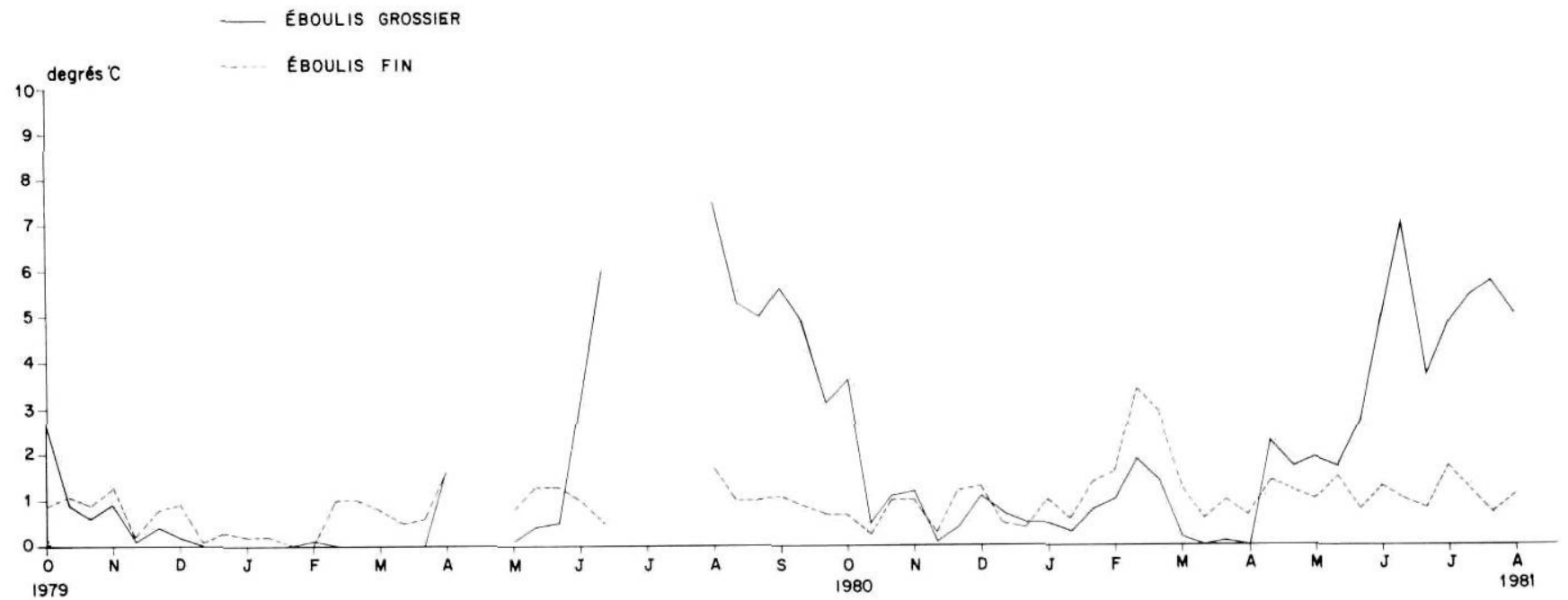

FIGURE 7. Amplitudes thermiques moyennes en adret $(2550 \mathrm{~m})$ dans le sol à $-50 \mathrm{~cm}$.

Average thermic ranges on the southern slope $(2550 \mathrm{~m})$ in the ground at $-50 \mathrm{~cm}$.

\section{TABLEAU I}

(G); les résultats d'une courte étude statistique sont résumés dans le tableau I.

Dans l'ensemble, les échanges thermiques en profondeur sont meilleurs qu'à l'ubac: près de $64 \%$ des amplitudes égalent ou dépassent $1^{\circ} \mathrm{C}$ contre $28 \%$ à l'ubac avec nettement plus de "grandes" amplitudes qu'à l'ubac $\left(22,4 \%\right.$ sont supérieures à $2^{\circ} \mathrm{C}$ contre $\left.5,5 \%\right)$. Cela est à mettre en relation avec l'exposition sud-est, la forte inclinaison de la pente $\left(34^{\circ}\right)$ et la minceur du manteau neigeux, lequel dépasse rarement $50 \mathrm{~cm}$, avec plusieurs périodes de "découverte" dans l'hiver par fusion de la neige avec des maxima absolus supérieurs

\begin{tabular}{lccccccc}
\hline $\begin{array}{l}\text { classe des amplitudes } \\
\text { matériel }\end{array}$ & \multicolumn{2}{c}{$A<0,5^{\circ} \mathrm{C}$} & \multicolumn{2}{c}{$A \geqslant 1^{\circ} \mathrm{C}$} & \multicolumn{2}{c}{$A>2^{\circ} \mathrm{C}$} \\
\hline $\begin{array}{l}\text { périodes } \\
\quad \text { été }\end{array}$ & & & & & & & \\
$\begin{array}{l}\text { 1er avril au } 30 \text { septembre } \\
\quad \text { hiver }\end{array}$ & 1,8 & 0 & 94,4 & 61 & 73,3 & 0 \\
$\begin{array}{l}\text { 1er octobre-31 mars } \\
\text { année }\end{array}$ & 33,2 & 13 & 39 & 61 & 5,4 & 1 \\
\hline & 17,5 & 6,5 & 66,7 & 61 & 39,3 & 5,5 \\
\hline
\end{tabular}

Amplitudes thermiques dans les éboulis grossiers et les éboulis fins de l'adret en été et en hiver (en \%) 
à $10^{\circ} \mathrm{C}$. La différence de comportement entre les deux types d'éboulis d'adret est donnée par la moyenne des amplitudes diurnes $(\bar{x})$ et, mieux encore, par l'écart type par rapport à cette moyenne $(\sigma)$, calculés entre le 1 er août 1980 et le 31 juillet 1981 :

$\begin{array}{ccc} & \begin{array}{c}\text { éboulis grossiers } \\ (-50 \mathrm{~cm})\end{array} & \begin{array}{c}\text { éboulis fins } \\ (-50 \mathrm{~cm})\end{array} \\ \overline{\mathrm{x}} & 2,5^{\circ} \mathrm{C} & 1,1^{\circ} \mathrm{C} \\ \sigma & 2,1^{\circ} \mathrm{C} & 0,6^{\circ} \mathrm{C}\end{array}$

En fait, c'est au cours de l'année que la différence apparaît bien :

a) Dans les blocs en open-work, trois périodes apparaissent (fig. 7): (1) une période où les amplitudes sont négligeables $(A<1)$, pendant laquelle stationne un manteau dépassant $30 \mathrm{~cm}$; (2) une période où elles sont fortes $(A>2)$ qui correspond aux mois d'été, avec déneigement total, des minima moyens positifs au fond de la combe et des maxima absolus dépassant $24^{\circ} \mathrm{C}$ à l'adret: (3) une période d'amplitudes moyennes voisines de $2^{\circ} \mathrm{C}$ où la couche de neige tombe en dessous de $30 \mathrm{~cm}$; c'est le cas en février 81 et en avril 80 et 81 où les alternances peuvent se faire autour de $0^{\circ} \mathrm{C}$ dans un matériel imbibé d'eau.

b) Dans les graviers à matrice, la faiblesse de l'écart type montre une très forte concentration des valeurs autour d'une moyenne faible $\left(1,1^{\circ} \mathrm{C}\right)$; elle est maximale en été, alors que c'est l'hiver qui totalise les plus grands écarts : (1) d'une part pendant les périodes à fort enneigement où l'amplitude devient négligeable comme dans les blocs; (2) d'autre part pendant les épisodes de fusion du manteau en hiver (février 80), l'amplitude dépassant alors celle mesurée dans les blocs.

De telles différences suggèrent deux types d'échanges thermiques en fonction de la présence ou de l'absence du manteau neigeux:

a) Sans neige, les échanges d'air turbulent sont importants entre les blocs sans matrice et rendent compte des fortes amplitudes, ce qui ne se produit pas dans les fines particules en contact les unes avec les autres; d'ailleurs l'apport calorique à $-50 \mathrm{~cm}$ se fait au cours de la journée avec deux heures de décalage dans les premiers contre douze heures dans les secondes, sur la plus grande partie de la surface.

b) Avec neige, un matelas d'air froid intersticiel isolant se forme entre les blocs empêchant les échanges; tandis que les produits fins enregistrent mieux les variations de la surface, particulièrement en période de fusion hivernale du manteau, sous l'action des eaux de fusion qui percolent vers cet horizon; d'ailleurs, le décalage sur la surface est alors bien moindre qu'en été, soit quatre heures tout au plus.

On voit donc que malgré le manteau neigeux, les échanges thermiques ne sont pas nuls durant la saison froide dans la tranche supérieure des sols jusqu'à $50 \mathrm{~cm}$, notamment sur ces talus d'éboulis raides et filtrants: les variations restent sensibles en adret en hiver, mais elles sont fonction de la granulométrie des formations. Enfin, l'écoulement hypodermique des eaux à partir des parois proches assure sans doute une grande partie des échanges thermiques en profondeur. Il convient maintenant d'apprécier, à partir des données complètes d'une année, les températures atteintes par ces sols.

\section{LE REFROIDISSEMENT ET LE RÉCHAUFFEMENT DES SOLS AU COURS DE L'ANNÉE}

On parlera ici de réchauffement et de refroidissement du sol plutôt que d'engel ou de dégel, car les valeurs négatives enregistrées à cette profondeur sont souvent très faibles et par conséquent situées en deçà de la marge d'erreur de la mesure; de plus, on sait que le sol gèle la plupart du temps à une température un peu inférieure à $0^{\circ} \mathrm{C}$. II s'agit ici d'apprécier l'importance du stockage des "frigories" dans ces types de sol, et secondairement d'apporter quelques éléments sur la fréquence et l'intensité que peuvent y avoir les cycles de gel-dégel.

1 - Le stockage hivernal du froid à $-50 \mathrm{~cm}$

a) On note d'abord la faiblesse des températures négatives dans les sols du fond, à l'exception des croupes déneigées; avec moins d'un mètre de neige (fig. 2 et 4 ), on remarque la pénétration d'une onde négative dans le sol à pelouse déprimé (P3), alors qu'un gel trois fois plus intense est mesuré sur le replat venté $\left(-3^{\circ} \mathrm{C}\right.$ en $\mathrm{P} 4$ avec $10 \mathrm{~cm}$ de neige jusqu'à la fin décembre); I'homothermie faiblement négative $\left(-0,3^{\circ} \mathrm{C}\right)$ est acquise avec plus d'un mètre de neige en P3, mais on l'a vue se maintenir avec $40 \mathrm{~cm}$ de neige fortement tassée par la déflation pendant les trois mois froids ${ }^{2}$.

b) Le gel progressif du sol d'éboulis d'ubac est accentué par le retard de la constitution du manteau neigeux (fig. 6). L'enneigement très médiocre jusqu'en mars permet la pénétration d'une onde de gel de $-2,5^{\circ} \mathrm{C}$ vers $50 \mathrm{~cm}$ et à son maximum (1re décade de mars), l'écart avec la proche surface $(-20 \mathrm{~cm})$ n'est que de $0,5^{\circ} \mathrm{C}$. Ce gel progressif a lieu avec des minima moyens de moins de $-10^{\circ} \mathrm{C}$, des minima absolus atteignant $-20^{\circ} \mathrm{C}$ et un manteau neigeux de $30-40 \mathrm{~cm}$. II est cependant difficile, en l'absence de sondages plus profonds, de situer le niveau atteint par l'isotherme $0^{\circ} \mathrm{C}$ dans ce site, mais il est vraisemblable qu'il s'arrête avant un mètre; un tel engel semble pourtant loin d'être garanti chaque année, comme en témoignent les données fragmentaires recueillies l'hiver précédent (79-80), au cours duquel le manteau s'est constitué vigoureusement fin d'écembre (tabl. II):

2. Selon LLIBOUTRY (1965), la perméabilité de la couche de neige peut diminuer fortement en raison de l'apparition de strates horizontales plus denses dues aux métamorphoses successives qui réduisent la circulation de l'air. 


\section{TABLEAU ॥}

Températures recueillies mensuellement à la station U1 pendant la période "homothermique" (janvier-juillet) en 1980

\begin{tabular}{ccc}
\hline 1980 & $\mathrm{U} 1-20 \mathrm{~cm}$ & $\mathrm{U} 1-50 \mathrm{~cm}$ \\
\hline 15 janvier & $-0,8^{\circ} \mathrm{C}$ & $-0,3^{\circ} \mathrm{C}$ \\
15 février & $-0,1^{\circ} \mathrm{C}$ & $+0,03^{\circ} \mathrm{C}$ \\
15 avril & $-0,4^{\circ} \mathrm{C}$ & $-0,09^{\circ} \mathrm{C}$ \\
15 mai & $-0,8^{\circ} \mathrm{C}$ & $-0,4^{\circ} \mathrm{C}$ \\
15 juin & $-0,02^{\circ} \mathrm{C}$ & $+0,3^{\circ} \mathrm{C}$ \\
15 juillet & $-0,5^{\circ} \mathrm{C}$ & $0,0^{\circ} \mathrm{C}$ \\
28 juillet & $0,0^{\circ} \mathrm{C}$ & $0,0^{\circ} \mathrm{C}$ \\
\hline
\end{tabular}

Ainsi, abstraction faite des températures observées, I'hiver à enneigement tardif enregistre une intensité de gel à $-50 \mathrm{~cm}$ sensiblement supérieure à celle de l'hiver à enneigement plus important et précoce (rapport de 1 à 6 à la valeur minimale de froid atteinte). Cette différence inter-annuelle semble d'ailleurs la règle; en site de col, 300 mètres plus haut, au Pas de la Souvagea ${ }^{3}$, des mesures au même site ont donné à $-50 \mathrm{~cm}:-9^{\circ} \mathrm{C}$ entre septembre 1975 et le 27 août $1976 ;-1^{\circ} \mathrm{C}$ entre septembre 1976 et août 1978. Là aussi, c'est l'enneigement qui a fait la différence.

c) On met en évidence la faible pénétration du gel dans les éboulis d'adret, mais on voit apparaître de sensibles différences selon la granulométrie (fig. 8): (1) Dans les éboulis à blocs, le refroidissement hivernal est rapide et l'évolution thermique à l'échelle de la décade est répercutée correctement en profondeur, bien que très amortie, jusqu'à février; cela prouve qu'une couche neigeuse peu épaisse (rarement plus de $50 \mathrm{~cm}$ avant le début de mars) peut amortir totalement les amplitudes diurnes, mais laisser passer les ondes majeures en les tassant toutefois fortement; les températures négatives ne parviennent pas en effet à pénétrer durablement en profondeur. Ainsi, que ce soit en régime d'ouverture du manteau en février par suite de fusion ou d'épaississement au-delà de $30 \mathrm{~cm}$ (mars), la moyenne thermique reste proche de $0^{\circ} \mathrm{C}$. (2) Dans les matériaux plus fins ${ }^{4}$, on observe au contraire une inertie plus grande au refroidissement hivernal $\left(\right.$ le $0^{\circ} \mathrm{C}$ est atteint avec un mois de retard); de surcroît, les températures restent nettement positives pendant le minimum thermique de surface (janvier-février), alors qu'elles deviennent négatives quand se reconstitue en mars le manteau neigeux. En l'absence de mesures tout au long du profil, on peut

3. Données recueillies par les équipes du laboratoire de Géomorphologie du C.N.R.S. de Caen et de l'institut de Géographie d'Aix-en-Provence (1980).

4. II s'agit de graviers, limons et surtout des sables grossiers: il faudrait rappeler (expériences du Laboratoire du C.N.R.S. de Caen) que les sables à l'engel donnent rapidement un béton de glace sans gonflements, tandis que les limons, ici très minoritaires, gèlent lentement, par paliers successifs, avec de nombreuses ségrégations. néanmoins admettre que c'est l'ouverture du manteau en février qui a permis à une onde de froid de pénétrer en profondeur et de s'y maintenir ensuite pendant deux mois. Le déneigement temporaire de plein hiver pourrait ainsi geler en adret des éboulis fins sur plus de $50 \mathrm{~cm}$, mais pas des éboulis grossiers sans matrice. Soulignons par contre que par bon enneigement tout l'hiver en 1979-80, aucune température en dessous de $0^{\circ} \mathrm{C}$ n'a été mesurée à cette profondeur dans ces éboulis fins.

2- L'expulsion printanière et estivale du froid à $-50 \mathrm{~cm}$ (fig. 2, 6, 8)

Le mois d'avril en 1980 comme en 1981 marque l'arrivée des maxima moyens positifs en surface et le début de la fusion du manteau neigeux au moment où il vient d'atteindre son épaisseur maximale. II va être intéressant de voir si le maintien du manteau jusqu'à l'été prolonge les températures négatives dans les sols.

a) Dans le fond de la combe, on observe la fin du régime homothermique d'hiver à $0^{\circ} \mathrm{C}$ le jour même où la neige disparaît (la transmission de la chaleur est alors immédiate vers $50 \mathrm{~cm}$ dans ce sol saturé d'eau); on constate que de tous les sites étudiés, c'est celui dont le déneigement est le plus tardif (P3) qui passe le dernier aux températures positives (tabl. III):

\section{TABLEAU III}

Date du passage aux températures positives en quatre stations en 1981 et 1980

\begin{tabular}{|c|c|c|c|}
\hline Stations $(-50 \mathrm{~cm})$ & adret & base $(\mathrm{P} 3)$ base $(\mathrm{P} 4)$ & ubac \\
\hline \multicolumn{4}{|l|}{$\begin{array}{l}\text { Décade du passage } \\
\text { au dessus de } 0^{\circ} \mathrm{C}\end{array}$} \\
\hline - en 1981 & $3^{e}$ de mai & $2^{e}$ de juin $3^{e}$ de mai & $1^{\text {re }}$ de juin \\
\hline - en 1980 & $1^{\text {re }}$ de juin & $\begin{array}{l}3^{\mathrm{e}} \mathrm{de} \\
\text { juillet }\end{array}$ & $\begin{array}{l}3^{e} \text { de } \\
\text { juillet }\end{array}$ \\
\hline
\end{tabular}

Inversement, le site le plus vite déneigé (P4) dégèle quinze jours avant, bien que la température atteinte au printemps y soit plus basse (fig. 2). La pénétration de la chaleur estivale longtemps différée par l'écran nival est foudroyante dans ces sites de fond $\left(10^{\circ} \mathrm{C}\right.$ de hausse en un mois et demi) ce qui laisse peu de chance à un gélisol de se maintenir à cette altitude.

b) On peut faire la même remarque à l'adret où les éboulis, même les fins, suivent une fois libérés de la neige, la même pente positive que l'air, ${ }^{5}$ mais avec près d'un mois d'avance sur le fond de la combe.

c) En ubac, avec des maxima possibles dans l'air de près de $20^{\circ} \mathrm{C}$ dès la mi-mai, le dégel est acquis rapidement, avant le fond enneigé de la combe. Par contre le mouvement positif est amorcé dès mars à la faveur d'un

5. Il faut tenir compte cependant du fait que le tiers supérieur d'un éboulis d'adret déneige plus vite que sa base qui tend, elle, à s'aligner sur le modèle du fond de la combe. 


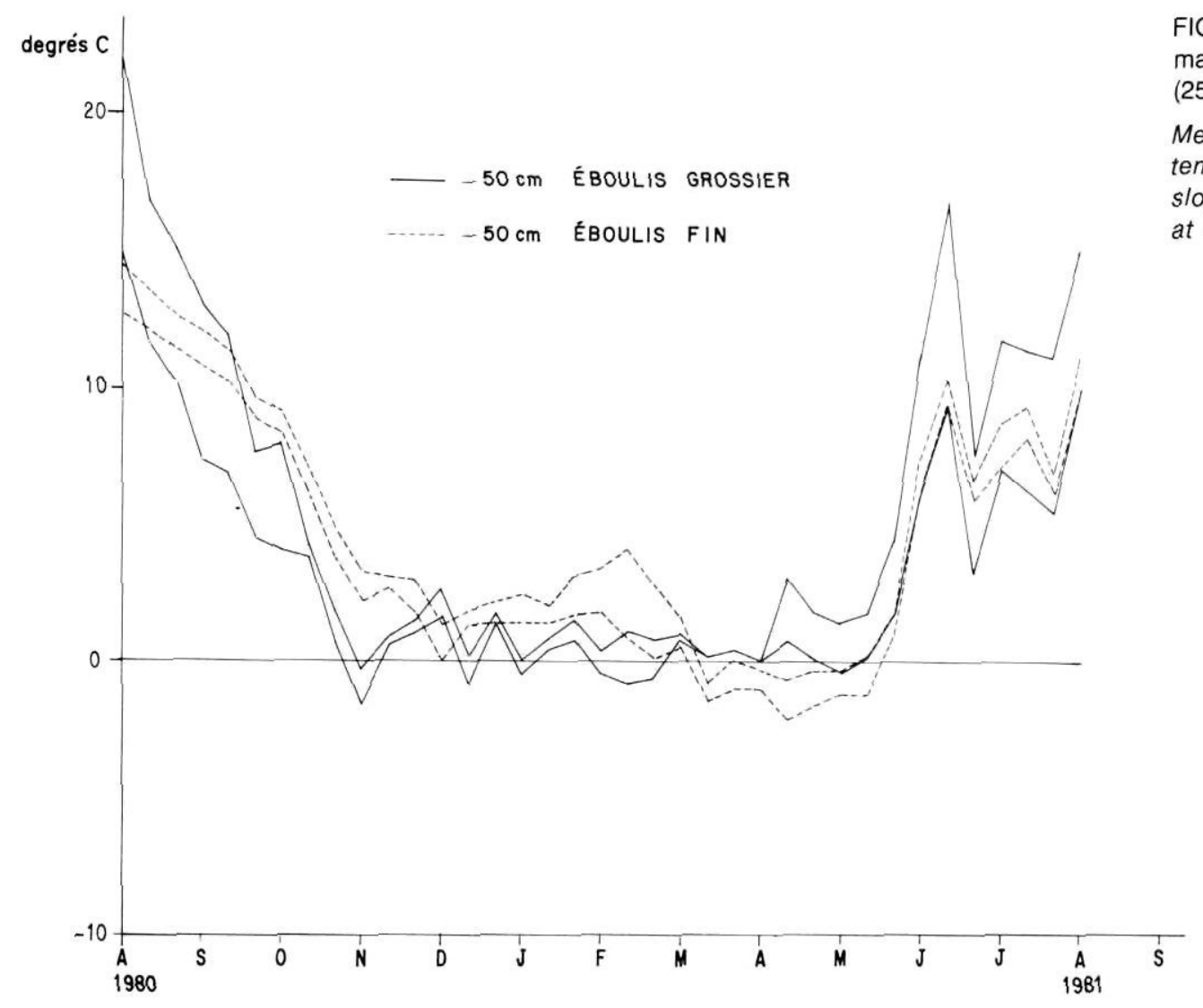

FIGURE 8. Moyennes des maxima et des minima en adret $(2550 \mathrm{~m})$ dans le sol à $-50 \mathrm{~cm}$.

Mean maximum and minimum temperatures on the southern slope $(2550 \mathrm{~m})$ in the ground at $-50 \mathrm{~cm}$.

début de fusion en paroi avec des maxima possibles de $+10^{\circ} \mathrm{C}$. Le dégel plus rapide de la partie inférieure du sol doit être mis là aussi en relation avec la percolation des eaux "chaudes" venant de la paroi bien ensoleillée en fin d'après-midi ${ }^{6}$. On notera avec intérêt qu'au printemps 1980 sur le même site, la plus longue persistance du manteau (un mois de plus) a permis la conservation entre $-20 \mathrm{~cm}$ et $-50 \mathrm{~cm}$ de températures voisines de $0^{\circ} \mathrm{C}$ jusqu'au 28 juillet avec des valeurs en surface proches de celles de 1981 (tabl. II et III).

d) A proximité de ce site, dans la rimaye thermique du névé permanent (station U3), les données recueillies au fond, vers $-500 \mathrm{~cm}$ de la surface, sont indiquées au tableau IV pour les deux années.

\section{TABLEAU IV}

Températures mesurées entre septembre 1979 et août 1981 au fond d'une rimaye thermique (station U3,2650 m)

\begin{tabular}{rrrrrr}
\hline & fin septembre & octobre & novembre janv.-mars & août \\
$1979-80$ & $-1,0^{\circ} \mathrm{C}$ & $+0,5^{\circ} \mathrm{C}$ & $-0,3^{\circ} \mathrm{C}$ & $-0,3^{\circ} \mathrm{C}$ & $0,0^{\circ} \mathrm{C}$ \\
$1980-81$ & $-1,8^{\circ} \mathrm{C}$ & $-0,3^{\circ} \mathrm{C}$ & $-1,0^{\circ} \mathrm{C}$ & $-0,5^{\circ} \mathrm{C}$ & $-0,5^{\circ} \mathrm{C}$ \\
\hline
\end{tabular}

6. L'ensoleillement est fort en fin de journée à la fin du printemps sur une paroi exposée au nord-ouest: l'apport calorique direct de la radiation s'ajoute alors à la chaleur reçue antérieurement par advection.
Pendant les mois mesurés, l'amplitude diurne n'a jamais dépassé en moyenne $0,5^{\circ} \mathrm{C}$. La température est restée toujours faiblement négative, avec un minimum net en automne quand la rimaye est bien ouverte. La forte masse neigeuse tendrait donc à maintenir, en profondeur au contact de la paroi, une homothermie légèrement négative tout au long de l'année.

Malgré cette exception, l'été (juillet-août) marque un net réchauffement de tous les sols; celui-ci est cependant inégal selon les sites, variant de $+6^{\circ} \mathrm{C}$ à $+20^{\circ} \mathrm{C}$. II apparaît ainsi que ce réchauffement est moins lié directement au franchissement d'un seuil thermique de l'air en surface qu'à celui d'une limite d'épaisseur dans le manteau neigeux.

\section{CONCLUSIONS}

A l'intérieur d'un espace restreint et dans une tranche altimétrique limitée à $200 \mathrm{~m}$, les régimes thermiques des sols étudiés dans la combe de Laurichard présentent des aspects très contrastés. Les influences de la topographie (exposition, pente), de la nature des matériaux et du régime d'enneigement au sol se combinent étroitement pour contrôler les échanges thermiques; il importe donc par conséquent de multiplier les points de mesures avant de tenter des généralisations sous forme de modèles. Néanmoins on peut risquer une première synthèse, en prenant en compte les mesures de deux années pendant lesquelles 
les manteaux neigeux ont connu une évolution bien différente.

On constate que le maintien des températures négatives en permanence, pendant près des cinq mois d'hiver dans l'air, ne suffit pas à constituer un niveau gelé en profondeur qui résiste à une saison "chaude» de quatre mois; sans doute les hivers ne sont-ils pas assez froids avec des températures moyennes de $-6,6^{\circ} \mathrm{C}$ (79-80) et de $-5,1^{\circ} \mathrm{C}(80-81)$, valeurs nettement supérieures à celles des climats des hautes latitudes conservant un pergélisol; sans doute aussi la neige empêche-t-elle le froid de s'accumuler durablement et profondément dans le sol. Néanmoins, on a vu que le régime du manteau au sol peut permettre certaines années la mise en place d'un gélisol durable: (1) lorsque son installation est retardée au-delà du minimum thermique vers le printemps, l'engel pouvant alors se produire dans les éboulis d'ubac, les éboulis fins d'adret et les croupes ventées du fond sur $50 \mathrm{~cm}$ au moins, si les teneurs en eau sont suffisantes au début de l'hiver; (2) lorsque son maintien au sol se prolonge vers le cœur de la saison chaude, ce qui n'est possible sur de grandes surfaces qu'en certains sites d'ubac.

Enfin, le réchauffement des sols en été a paru partout très net; il est lié directement à la disparition totale de la couverture neigeuse. Inversement, leur maintien sous la neige en cette saison peut les conserver à cette altitude à une température légèrement négative comme le suggèrent les températures de la rimaye thermique.

De telles constatations posent le problème de l'origine de la glace, intersticielle ou non, conservée dans les matériaux qui fluent actuellement sur l'ubac de la combe, dans les talus d'éboulis et les glaciers rocheux entre $2450 \mathrm{~m}$ et $2700 \mathrm{~m}$.

\section{DISCUSSION}

I- Les régimes thermiques actuels peuvent-ils être à l'origine des formations de glaces qui jouent un rôle important sur la morphogenèse des ubacs à partir de $2500 \mathrm{~m}$ dans les Alpes internes?

En l'absence de mesures prises au pied des talus et sur les racines des glaciers rocheux, on peut concevoir, à la lumière de ce qui précède, les conditions les plus favorables susceptibles de constituer ou d'entretenir des stocks de glaces dans les matériaux éboulisés :

a) Une combinaison optimale au cours de l'année des régimes thermiques et nivométriques, laquelle nous paraît être: des hivers froids et secs dont le déficit en neige jusqu'à mars est accentué par une déflation continue $^{7}$, suivis de printemps humides et frais gardant le

7. Une telle condition se retrouve dans la formule empirique de Schostakowitch datant de 1927 (citée par J. TRICART 1967) posant que le tjaële ne peut se former quand l'épais- manteau neigeux jusqu'au cœur de l'été ; des étés sans chaleur maintenant de larges taches de neige résiduelles, vite interrompus par des automnes précoces présentant de fréquentes chutes de neige fondant régulièrement pour fournir une charge en eau suffisante aux matériaux à l'entrée de l'hiver.

b) Des conditions topographiques particulières: Un gélisol prolongé dans des éboulis d'adret et sur les fonds de vallon avec pelouse ou lande à cette altitude est exclu; par contre, les pieds d'éboulis enrichis en éléments fins et riches en eau, longtemps recouverts par les culots d'avalanches, peuvent offrir à partir de 2600-2700 m des sites favorables, surtout en exposition nord à nord-est. Des ségrégations de glace formées en hiver peuvent s'y maintenir en été, grâce notamment au caractère endothermique de la fusion. Mais ne disposant pas ici de données pouvant confirmer l'existence de tels processus, on est obligé de rappeler les autres origines possibles de ces glaces: Le recouvrement des névés saisonniers par les produits gélifractés provenant de la paroi en voie de démantèlement rapide, en les intégrant lentement sous forme de glace aux matériaux, processus qui a bien été observé par LLIBOUTRY (1965) dans les Andes sèches et par nous même dans les Alpes internes; la persistance d'une masse glaciaire héritée dont l'ensevelissement sous les tabliers d'éboulis prolonge la décrépitude depuis le Petit Âge glaciaire, avec l'appoint éventuel de l'onde de froid hivernal et le regel des eaux de percolation.

Nous avons montré à plusieurs reprises (FRANCOU, 1981, 82, 83), sans qu'il soit nécessaire d'y revenir ici, le rôle de ces glaces enterrées dans la morphologie des éboulis "fluants" et la filiation évidente qui existe entre ces derniers et les glaciers rocheux qui viennent s'y enraciner.

II-Quelle est l'efficacité des cycles de gel-dégel dans ces sols?

La cryoclastie opère en surface, comme en témoignent les nombreux blocs éclatés dont les fragments s'emboîtent, particulièrement dans les matériaux qui ont subi au préalable des altérations hydrothermales poussées; elle est responsable aussi d'un émoussé de désagrégation granulaire dans les granites, lequel se forme rapidement, en quelques siècles sur les matériaux immobilisés. Par contre, en profondeur, la faiblesse des amplitudes enregistrées autour de $0^{\circ} \mathrm{C}$, qu'elles soient diurnes ou saisonnières, limite probablement la fragmentation, même si la charge en eau des matériaux est plus élevée à ce niveau. Plus près de la surface, l'efficacité du gel doit augmenter sensiblement, surtout en adret en période hivernale et automnale quand la couche de neige s'amincit en deçà de $30 \mathrm{~cm}$. Mais il faudra

seur du manteau neigeux mesurée fin janvier est supérieure au double de la somme des valeurs absolues des moyennes mensuelles négatives de la température. 
recueillir les températures sur tout le profil entre $-50 \mathrm{~cm}$ et la surface pour apporter là-dessus des éléments plus précis.

\section{REMERCIEMENTS}

Je remercie ici MM. O. Dollfus (université de Paris VII), Y. Bravard (université de Grenoble 1), H. Cortot, du parc national des Écrins, d'avoir prêté un concours indispensable aux campagnes de mesures et d'avoir ainsi permis que cette étude voit le jour. MM. J.P. Coutard et J.P. Lautridou ont bien voulu relire ces lignes et me faire part de leurs commentaires. Les professeurs Ch. P. Péguy et P. Paul m'ont aidé, fort courtoisement, a corriger le manuscrit.

\section{RÉFÉRENCES}

C.N.R.S. CAEN et UNIVERSITÉ D'AIX-EN-PROVENCE (1980): Obsenations sur quelques formes et processus périglaciaires dans le massif du Chambeyron (Alpes de HautesProvence), Revue de géographie alpine, t. LXVIII, $n^{\circ} 4$, p. 349-382.
FRANCOU, B. (1981): Géodynamique des éboulis et dépôts associés de la combe de Laurichard (Hautes-Alpes), thèse de $3^{e}$ cycle, Grenoble, 230 p. (non publ.)

- (1982): Chutes de pierres et éboulisation dans les parois de l'étage périglaciaire, Revue de géographie alpine, t. LXX, n० 3, p. 279-300.

(1983): Géodynamique des dépôts de pied de paroi dans l'étage périglaciaire (à paraître).

JORDA, M. (1978): Les formations à blocs des milieux subalpins et alpins des Alpes de Haute Provence, Colloque sur le périglaciaire d'altitude du domaine méditerranéen et abords, Strasbourg, p. 31-44.

LAUTRIDOU, J.P. (1981): Conclusions générales des expériences de gélifraction expérimentale, C.N.R.S., Caen, $n^{\circ} 21$ p. $65-84$.

LLIBOUTRY, L. (1965): Traité de glaciologie, Paris, Masson, $1040 \mathrm{p}$.

MUNN, R.E. (1966): Descriptive Micrometeorology, New York et Londres, Academic Press, $245 \mathrm{p}$.

THORN, C.E. (1980): Alpine bedrock temperatures: an empirical Study, Arctic and Alpine Research, vol. 12, $\mathrm{n}^{\circ} 1$, p. 73-86.

TRICART, J. et CAILLEUX, A. (1967): Le modelé des régions périglaciaires, Paris, S.E.D.E.S., 512 p. 